PROCEEDINGS OF THE AMERICAN MATHEMATICAL SOCIETY

Volume 124, Number 9, September 1996

\title{
TRANSFORMATIONS CONJUGATE TO THEIR INVERSES HAVE EVEN ESSENTIAL VALUES
}

\author{
GEOFFREY GOODSON AND MARIUSZ LEMAŃCZYK
}

(Communicated by Mary Rees)

\begin{abstract}
Let $T$ be an ergodic automorphism defined on a standard Borel probability space for which $T$ and $T^{-1}$ are isomorphic. We study the structure of the conjugating automorphisms and attempt to gain information about the structure of $T$. It was shown in Ergodic transformations conjugate to their inverses by involutions by Goodson et al. (Ergodic Theory and Dynamical Systems 16 (1996), 97-124) that if $T$ is ergodic having simple spectrum and isomorphic to its inverse, and if $S$ is a conjugation between $T$ and $T^{-1}$ (i.e. $S$ satisfies $T S=S T^{-1}$ ), then $S^{2}=I$, the identity automorphism. We give a new proof of this result which shows even more, namely that for such a conjugation $S$, the unitary operator induced by $T$ on $L^{2}(X, \mu)$ must have a multiplicity function whose essential values on the ortho-complement of the subspace $\left\{f \in L^{2}(X, \mu): f\left(S^{2}\right)=f\right\}$ are always even. In particular, we see that $S$ can be weakly mixing, so the corresponding $T$ must have even maximal spectral multiplicity (regarding $\infty$ as an even number).
\end{abstract}

\section{INTRODUCTION}

Let $T$ be an ergodic transformation defined on a standard Borel probability space $(X, \mathcal{F}, \mu)$ having simple spectrum. It was shown in [2] that if $T$ is isomorphic to its inverse $T^{-1}$ and the conjugating automorphism is $S$, i.e. $T S=S T^{-1}$, then $S^{2}=I$, the identity automorphism. It follows that if $T$ has a conjugating automorphism $S$ for which $S^{2} \neq I$, then $T$ has non-simple spectrum. Our aim is to obtain more information about the nature of the spectrum in this situation. In this paper we show that in fact the spectrum of $T$ must have even multiplicity in the orthogonal complement of the subspace $\left\{f \in L^{2}(X, \mu): f\left(S^{2}\right)=f\right\}$ (we regard $\infty$ as an even number). Of course this ortho-complement is trivial if $T$ has simple spectrum, and it consists of everything except the constant functions if $S$ is weak mixing. Specifically we prove

Theorem 1. Suppose that $S T=T^{-1} S$ for some automorphism $S$; then the essential values of the multiplicity function of $T$, restricted to the ortho-complement of the subspace

$$
\left\{f \in L^{2}(X, \mu): f\left(S^{2}\right)=f\right\}
$$

are even.

Received by the editors November 1, 1994 and, in revised form, February 27, 1995.

1991 Mathematics Subject Classification. Primary 28D05; Secondary 47A35.

The second author was partially supported by a KBN grant.

(C)1996 American Mathematical Society 
This theorem, which is proved in section 3, actually follows from a more general result regarding unitary operators on $L^{2}$-spaces which preserve real-valued functions and are unitarily equivalent to their inverses.

In section 2 we show that if $S^{2}$ and $T$ with $S T=T^{-1} S$ are both ergodic, then they both must be weak mixing.

Finally, in the last section we give a number of examples illustrating the types of situations which may arise. In particular, examples for which $S$ is weak mixing are given.

\section{Preliminaries}

Throughout, $T:(X, \mathcal{F}, \mu) \rightarrow(X, \mathcal{F}, \mu)$ will denote an ergodic automorphism defined on a non-atomic standard Borel probability space. Throughout the identity automorphism will be denoted by $I$. The group of all automorphisms $\mathcal{U}(X)$ of $(X, \mathcal{F}, \mu)$ becomes a completely metrizable topological group when endowed with the weak convergence of transformations $\left(T_{n} \rightarrow T\right.$ if for all $A \in \mathcal{F}$, $\mu\left(T_{n}^{-1}(A) \triangle T^{-1}(A)\right)+\mu\left(T_{n}(A) \triangle T(A)\right) \rightarrow 0$ as $\left.n \rightarrow \infty\right)$. Denote by $C(T)$ the commutant of $T$, i.e. those automorphisms of $(X, \mathcal{F}, \mu)$ which commute with $T$. Since we are assuming that the members of $C(T)$ are invertible, $C(T)$ is a group. Suppose that $X$ is an atomic space; then, if $T: X \rightarrow X$ is ergodic, $T$ is a rotation on a finite point set and so is automatically isomorphic to $T^{-1}$ by an involution. Here the theory reduces to the theory of cyclic permutations, and consequently we always assume that $X$ is a non-atomic probability space.

Related to the commutant is the set

$$
\mathcal{B}(T)=\left\{S \in \mathcal{U}(X): T S=S T^{-1}\right\} .
$$

If $S$ is measure preserving and $T S=S T^{-1}$, then $S^{2} T=S T^{-1} S=T S^{2}$. This shows that $\left\{S^{2}: S \in \mathcal{B}(T)\right\} \subseteq C(T)$.

The spectral properties of $T$ are those of the induced unitary operator defined by

$$
U_{T}: L^{2}(X, \mu) \rightarrow L^{2}(X, \mu) ; \quad U_{T} f(x)=f(T x), \quad f \in L^{2}(X, \mu) .
$$

Generally a unitary operator $U: H \rightarrow H$ on a separable Hilbert space $H$ is said to have simple spectrum if there exists $h \in H$ such that $Z(h)=H$, where $Z(h)$ is the closed linear span of the vectors $U^{n} h, n \in \mathbb{Z}$. $U$ is determined up to unitary equivalence by a spectral measure class $\sigma$ and a $\{1,2, \ldots, \infty\}$-valued multiplicity function $M$ defined on the circle $S^{1}$. The set $\mathcal{M}_{U}$ of essential spectral multiplicities of $U$ is the set of all $\sigma$-essential values of $M$. The maximal spectral multiplicity (or just multiplicity) of $U$ is $\operatorname{msm}(U)=\sup \mathcal{M}_{U}$. $U$ has simple spectrum if $\operatorname{msm}(U)=$ 1 , otherwise $U$ has non-simple spectrum. See Queffelec [7] for a detailed discussion of the spectral properties of dynamical systems.

\section{BASIC RESULTS}

In this section, we show that if $S \in \mathcal{B}(T)$ where both $T$ and $S^{2}$ are ergodic, then they must both be weak mixing. This leads to a simple classification of the members of $\mathcal{B}(T)$. The first part of the next proposition was given in [2]. The second part is well known, consequently we omit their proofs, both being elementary and easy.

Proposition 1. Let $T: X \rightarrow X$ be an ergodic automorphism.

(i) If $S \in \mathcal{B}(T)$, then $S^{2 n}=I$ for some $n>0$, or $S$ is aperiodic. 
(ii) If $T S=S T$ where $T$ is weak mixing and $S$ is ergodic, then $S$ is also weak mixing.

Ryzhikov [8] improved on results of Fathi [1] by showing that any automorphism $T$ on a standard Borel probability space $(X, \mathcal{F}, \mu)$ is the product of three involutions (i.e. $T: X \rightarrow X$ can be written $T=U \circ V \circ W$ where $U^{2}=V^{2}=W^{2}=I$ ). This is best possible (it was mentioned in [2], and it is an elementary fact that $T$ is isomorphic to its inverse via an involution $S$, if and only if $T$ is the product of two involutions).

In this direction, below we give a simple characterization of transformations which are isomorphic to their inverses.

Proposition 2. An automorphism $T: X \rightarrow X$ is isomorphic to its inverse if and only if there exist automorphisms $S_{1}$ and $S_{2}$ for which $T=S_{1} \circ S_{2}^{-1}$ and satisfying $S_{1}^{2}=S_{2}^{2}$.

Proof. First suppose that $T$ is isomorphic to its inverse; then there exists $S_{1} \in \mathcal{B}(T)$ satisfying $S_{1} T=T^{-1} S_{1}$. Let $S_{2}=T^{-1} \circ S_{1}$; then $S_{2} \in \mathcal{B}(T)$ and

$$
S_{1} \circ S_{2}^{-1}=S_{1} \circ\left(T^{-1} \circ S_{1}\right)^{-1}=T,
$$

and

$$
S_{2}^{2}=\left(T^{-1} \circ S_{1}\right)^{2}=T^{-1} \circ S_{1} \circ T^{-1} \circ S_{1}=S_{1} \circ T \circ T^{-1} \circ S_{1}=S_{1}^{2} .
$$

Conversely, suppose that $T=S_{1} \circ S_{2}^{-1}$, where $S_{1}^{2}=S_{2}^{2}$; then

$$
S_{1} \circ T=S_{1} \circ\left(S_{1} \circ S_{2}^{-1}\right)=S_{1}^{2} \circ S_{2}^{-1}=S_{2},
$$

and similarly, $T^{-1} \circ S_{1}=S_{2}$, so that $S_{1} \in \mathcal{B}(T)$ and $T$ is isomorphic to its inverse.

The following simple corollary shows that if $T$ is ergodic, the members of $\mathcal{B}(T)$ never commute, and if $S \in \mathcal{B}(T)$, then $S^{2}$ has non-simple spectrum. This is mainly of interest when $S$ is also ergodic.

Corollary 1. If $T$ is ergodic and $S \in \mathcal{B}(T)$, then $C\left(S^{2}\right)$, the commutant of $S^{2}$, is non-abelian. In particular, $S^{2}$ has a non-simple spectrum.

Proof. Suppose there exists $S_{1} \in \mathcal{B}(T)$ for which $C\left(S_{1}^{2}\right)$ is abelian. If we define $S_{2}=S_{1} \circ T$, we see that $S_{1}^{2}=S_{2}^{2}$.

Thus the map $R=S_{1}^{2}=S_{2}^{2}$ has 2 distinct square roots $S_{1}, S_{2}$, which therefore belong to $C(R)$ and hence commute.

Now $T=S_{1}^{-1} \circ S_{2}$, so that

$$
T^{2}=S_{1}^{-1} \circ S_{2} \circ S_{1}^{-1} \circ S_{2}=S_{1}^{-2} \circ S_{2}^{2}=I,
$$

contradicting $T$ being ergodic.

We now show that if $T$ is ergodic and $S \in \mathcal{B}(T)$ where $S^{2}$ is ergodic, then $T$ has to be weak mixing, which in turn implies that $S$ is weak mixing. In a later section we shall give a number of examples of automorphisms conjugated to their inverses by weak mixing transformations.

Theorem 2. Suppose that $T: X \rightarrow X$ and $S: X \rightarrow X$ are automorphisms for which $T$ and $S^{2}$ are ergodic and $S T=T^{-1} S$. Then both $S$ and $T$ are weak mixing. 
Proof. Suppose that $f(T x)=\lambda f(x)$ for some measurable function $f$ and constant $\lambda \in S^{1}$. Then

$$
f(T S x)=\lambda f(S x), \text { so that } f\left(S T^{-1} x\right)=\lambda f(S x) .
$$

This implies that $\bar{\lambda} f(S x)=f(S T x)$, or that $f \circ S(T x)=\bar{\lambda} f \circ S(x)$.

Thus $\bar{\lambda}$ is an eigenvalue of $T$ corresponding to the eigenfunction $f \circ S$. In particular

$$
\bar{f} \circ S(T x)=\lambda \bar{f} \circ S(x) .
$$

The ergodicity of $T$ implies that $\bar{f} \circ S=c \cdot f$ for some constant $c$, with $|c|=1$. Then

$$
f\left(S^{2} x\right)=f \circ S(S x)=\bar{c} \bar{f}(S x)=\bar{c} \cdot c f(x)=f(x) .
$$

But $S^{2}$ is ergodic, so $f=$ constant and $\lambda=1$, so that $T$ is weak mixing. Proposition 1 now implies that $S^{2}$ and hence $S$ are weak mixing.

Corollary 2. Let $S \in \mathcal{B}(T)$ for some ergodic $T$.

(i) If $f$ is an eigenfunction for $T$ with corresponding eigenvalue $\lambda$, then $f \circ S$ is an eigenfunction corresponding to the eigenvalue $\bar{\lambda}$.

(ii) Let $H_{0}$ be the closed linear span of the eigenfunctions of $T$. If for any $S \in \mathcal{B}(T), H_{S}=\left\{f \in L^{2}(X, \mu): S^{2}(f)=f\right\}$, then $H_{0}$ and $H_{S}$ are both $T$ and $S$ invariant subspaces of $L^{2}(X, \mu)$ which satisfy $H_{0} \subseteq H_{S}$.

Proof. (i) follows directly from the last theorem.

(ii) can be proved directly, or alternatively we can argue as follows:

Denote by $S_{0}=S \mid H_{0}$ and $T_{0}=T \mid H_{0}$. Then $T_{0}: H_{0} \rightarrow H_{0}$ has discrete spectrum and hence has simple spectrum. Furthermore $S_{0} T_{0}=T_{0}^{-1} S_{0}$ and the conditions of Theorem 1 of [2] are satisfied. (In particular, $H_{0}$ may be represented as $L^{2}(Y)$ for suitable $Y$ corresponding to the maximal Kronecker factor of $T$.) This implies that $S_{0}^{2}=I$ and the result follows.

Corollary 2 gives another proof that if $S^{2}$ is ergodic, then $T$ is weak mixing (and hence $S$ is weak mixing). This is because the ergodicity of $S^{2}$ implies that $H_{S}$ is the one-dimensional subspace of $L^{2}(X, \mu)$ consisting only of the constant functions. The same therefore applies to $H_{0}$ and the result follows.

The following corollary is now an immediate consequence of Theorem 2 and Proposition 1. The quasi-discrete spectrum transformations of [2] give rise to examples for which (iii) below holds.

Corollary 3. For each $S \in \mathcal{B}(T)$, one of the following must hold:

(i) $S^{2 n}=I$ for some $n \geq 1$,

(ii) $S$ is weakly mixing,

(iii) $S^{2}$ is aperiodic and non-ergodic.

\section{Conjugation by a unitary operator}

In this section we attempt to explain why all known ergodic automorphisms having a finite Lebesgue component in their spectrum have Lebesgue components of even multiplicity. (See the examples of Mathew-Nadkarni and Lemańczyk, where the Lebesgue component in the spectrum arises in the ortho-complement of the eigenfunctions.) In particular, we show that for a unitary operator $T: L^{2}(X, \mu) \rightarrow$ 
$L^{2}(X, \mu)$, preserving real-valued functions, and $S \in \mathcal{B}(T)$ (also preserving realvalued functions), $T$ has a multiplicity function which only takes even values (a.e.) in the ortho-complement of the subspace $\left\{f \in L^{2}(X, \mu): S^{2}(f)=f\right\}$. It follows that if $T: X \rightarrow X$ is an automorphism having simple spectrum and $S \in \mathcal{B}(T)$, then $S^{2}=I$, giving a new proof of Theorem 1 of [2].

The following is the main theorem of the paper, and gives Theorem 1 as an immediate consequence. In this case $\mathcal{B}(T)$ denotes the unitary conjugations between $T$ and $T^{-1}$.

Theorem 3. Suppose that $T: L^{2}(X, \mu) \rightarrow L^{2}(X, \mu)$ is a unitary operator which preserves real-valued functions, and suppose there exists $S \in \mathcal{B}(T)$ which also preserves real-valued functions. Then in the ortho-complement of the subspace

$$
\left\{f \in L^{2}(X, \mu): S^{2}(f)=f\right\},
$$

the essential values of the multiplicity function of $T$ are even $(\infty$ is considered as an even number).

Proof. Let $H_{1}=\left\{f \in L^{2}(X, \mu): S^{2}(f)=f\right\}, H_{-1}=\left\{f \in L^{2}(X, \mu): S^{2}(f)=-f\right\}$ and $H=H_{1} \oplus H_{-1}$; then since $S T=T^{-1} S, H_{1}$ and $H_{-1}$ are both $T$ and $S$ invariant, so the same is true for $H^{\perp}$.

We shall see that $T$ preserves $\mathcal{P}_{1}$ and $\mathcal{P}_{2}$ where

$$
H^{\perp}=\mathcal{P}_{1} \oplus \mathcal{P}_{2},
$$

and where $\mathcal{P}_{1}=$ those functions whose spectral type is concentrated on the "upper" half, $S^{+}$, of the unit circle in the complex plane (excluding \pm 1 ), and $\mathcal{P}_{2}=$ those whose spectral type is concentrated on the "lower" half $S^{-}$(again excluding \pm 1 ) of the unit circle (spectral type here means with respect to $S^{2}$ ). More precisely, let

$$
\begin{aligned}
& \mathcal{P}_{1}=\left\{f \in H^{\perp}: \operatorname{supp} \sigma_{f} \subseteq S^{+}\right\}, \\
& \mathcal{P}_{2}=\left\{f \in H^{\perp}: \operatorname{supp} \sigma_{f} \subseteq S^{-}\right\}
\end{aligned}
$$

(where supp $\sigma_{f}$ denotes the support of the measure $\sigma_{f}$, the spectral type of $f$ with respect to $S^{2}$ ). Since $S^{2}$ preserves real-valued functions, it also preserves complex conjugation (i.e. $S^{2}(\bar{f})=\overline{S^{2}(f)}$ for all $f \in L^{2}(X, \mu)$ ). It follows that on $H^{\perp}$, the maximal spectral type of $S^{2}$ is invariant under complex conjugation, so if $h$ realizes its maximal spectral type, we can define measures $\nu_{1}$ and $\nu_{2}$ on $S^{1}$ by

$$
\nu_{1}(A)=\sigma_{h}\left(A \cap S^{+}\right), \quad \nu_{2}(A)=\sigma_{h}\left(A \cap S^{-}\right),
$$

and since $\sigma_{h}$ is symmetric on $S^{1}$,

$$
\nu_{1}(\bar{A})=\sigma_{h}\left(\bar{A} \cap S^{+}\right)=\sigma_{h}\left(\overline{A \cap S^{-}}\right)=\sigma_{h}\left(A \cap S^{-}\right)=\nu_{2}(A) .
$$

Now $\nu_{i} \ll \sigma_{h}, i=1,2$, implies that there exists $h_{1}$ and $h_{2}$ with $\nu_{1}=\sigma_{h_{1}}, \nu_{2}=\sigma_{h_{2}}$. It follows that $\nu_{2}=\sigma_{\bar{h}_{1}}$, where

$$
h=h_{1}+\bar{h}_{1},
$$

and where $h_{1} \in \mathcal{P}_{1}, \bar{h}_{1} \in \mathcal{P}_{2}$. Therefore

$$
\begin{aligned}
& \mathcal{P}_{1}=\left\{f \in H^{\perp}: \sigma_{f} \ll \sigma_{h_{1}}\right\}, \\
& \mathcal{P}_{2}=\left\{f \in H^{\perp}: \sigma_{f} \ll \sigma_{\bar{h}_{1}}\right\} .
\end{aligned}
$$


Here the spectral type is with respect to $S^{2}$, and it is clear that these subspaces are $S^{2}$ and $T$ invariant and also that equation (1) holds.

Now we claim that

$$
T: \mathcal{P}_{1} \rightarrow \mathcal{P}_{1} \text { and } T: \mathcal{P}_{2} \rightarrow \mathcal{P}_{2}
$$

are unitarily equivalent.

First note that if $f \in \mathcal{P}_{1}$, then $S(\bar{f}) \in \mathcal{P}_{2}$ and

$$
\begin{aligned}
& \left(T^{n} f, f\right)=\left(S T^{n} f, S f\right)=\left(T^{-n} S f, S f\right) \\
= & \left(S f, T^{n} S f\right)=\overline{\left(T^{n} S f, S f\right)}=\left(T^{n} S \bar{f}, S \bar{f}\right) .
\end{aligned}
$$

Consequently, if $Z(f)$ and $Z(S \bar{f})$ are the corresponding cyclic subspaces with respect to $T$, they are also subspaces of $\mathcal{P}_{1}$ and $\mathcal{P}_{2}$ respectively, so that $T \mid Z(f)$ and $T \mid Z(S \bar{f})$ are unitarily equivalent (this already shows that $T$ has non-simple spectrum when $H^{\perp}$ is non-trivial).

Now let

$$
\mathcal{P}_{1}=\bigoplus_{i=1}^{\infty} Z\left(k_{i}\right)
$$

be a spectral decomposition (with respect to $T$ ) for $T \mid \mathcal{P}_{1}$. Then

$$
\sigma_{k_{1}} \gg \sigma_{k_{2}} \gg \ldots \text {. }
$$

Now $S \bar{k}_{i} \in \mathcal{P}_{2}$ and $S Z\left(\bar{k}_{i}\right)=Z\left(S \bar{k}_{i}\right)$, for $i=1,2, \ldots$ Clearly

$$
\bigoplus_{i=1}^{\infty} Z\left(S \bar{k}_{i}\right)=\mathcal{P}_{2}
$$

with $\sigma_{S \bar{k}_{i}}=\sigma_{k_{i}}, i=1,2, \ldots$, and $\sigma_{S \bar{k}_{1}} \gg \sigma_{S \bar{k}_{2}} \gg \ldots$.

It follows that

$$
\bigoplus_{i=1}^{\infty} Z\left(S \bar{k}_{i}\right) \text { is a spectral decomposition for } T \mid \mathcal{P}_{2} .
$$

The unitary equivalence between $Z\left(k_{i}\right)$ and $Z\left(S \bar{k}_{i}\right)$ for $i=1,2, \ldots$, now gives the required result.

We now consider the case of $T \mid H_{-1}$. As before, if $f \in H_{-1}$, then $f$ and $S \bar{f}$ have the same spectral type, but in this case we also have $S \bar{f} \in H_{-1}$. Here we proceed as follows:

$$
\left(T^{n} f, S \bar{f}\right)=\left(S T^{n} f, S^{2} \bar{f}\right)=\left(T^{-n} S f,-\bar{f}\right)=-\left(S f, T^{n} \bar{f}\right)=-\left(T^{n} f, S \bar{f}\right) .
$$

It follows that $\left(T^{n} f, S \bar{f}\right)=0$ for all $n \in \mathbb{Z}$, so that as before, $T \mid Z(f)$ and $T \mid Z(S \bar{f})$ are unitarily equivalent, and similar arguments to those earlier complete the proof.

\section{EXAMPLES}

We now give two examples of automorphisms which have conjugations to their inverses which are weak mixing. Suppose that $R$ is a weak mixing transformation having two distinct square roots $S_{1}$ and $S_{2}$, i.e. $R=S_{1}^{2}=S_{2}^{2}$; then it follows from Proposition 2 that $\widehat{T}=S_{1} \circ S_{2}^{-1}$ is isomorphic to its inverse and $S_{1}, S_{2}$ are weak mixing conjugations. It may be that $\widehat{T}$ is not ergodic, for example, suppose $T$ is weakly mixing and $R=T^{2} \times T^{2}, S_{1}=T \times T$ and $S_{2}(x, y)=\left(y, T^{2} x\right)$. If 
$\widehat{T}(x, y)=S_{1} \circ S_{2}^{-1}(x, y)=\left(T^{-1} y, T x\right)$, then $\widehat{T}^{2}=I$. Below we give two examples giving rise to ergodic (and hence weak mixing) $\widehat{T}$.

(i) Let $T: X \rightarrow X$ be a weak mixing automorphism; then $T \times T$ is also weak mixing. Define $S: X \times X \rightarrow X \times X$ by $S(x, y)=(y, T x)$. Clearly $S$ is a square root of $T \times T$, so is weak mixing. Furthermore, $S$ conjugates $T \times T^{-1}$ to its inverse $T^{-1} \times T$, even if $T$ and $T^{-1}$ are non-isomorphic. This is the situation of the above remark where we have chosen $R=T \times T$, having the two distinct square roots, $S(x, y)=(y, T x)$ and $S^{\prime}(x, y)=(T y, x)$, so that $T \times T^{-1}=S^{\prime} \circ S^{-1}$.

In [3], del Junco and Lemańczyk have shown that there is a dense $G_{\delta}$ subset $\mathcal{K}$ of $\mathcal{U}(X)$ such that for each $T \in \mathcal{K}, C\left(T \times T^{-1}\right)=C(T) \times C(T)$. It follows from their results that for $T \in \mathcal{K}$

$$
\mathcal{B}\left(T \times T^{-1}\right)=\{(P \times Q) \circ F: P, Q \in C(T)\},
$$

where $F$ is the flip map, $F(x, y)=(y, x)$. Note that $\mathcal{B}\left(T \times T^{-1}\right)$ contains many involutions, in fact any map of the form $U(x, y)=\left(P y, P^{-1} x\right)$ is such an involution.

Remark. Theorem 3 implies that the essential values of $T \times T^{-1}$ are even for every $T \in \mathcal{U}(X)$, and since it is not hard to see that $T \times T$ is spectrally isomorphic to $T \times T^{-1}$, it follows that the essential values of $T \times T$ are always even. Katok [4] has shown that generically the essential values of $T \times T$ are either $\{2\}$ or $\{2,4\}$.

(ii) This next example was communicated to us by J. Feldman, and although somewhat similar to example (i), it is of interest because $\mathcal{B}(\widehat{T})$ contains maps of a different nature, namely types of shift maps.

We start with an ergodic automorphism $T$ of $(X, \mathcal{F}, \mu)$, a non-atomic standard Borel space. Let $(Y, \mathcal{B}, m)=\prod_{i=-\infty}^{\infty}(X, \mathcal{F}, \mu)_{i}$ be the doubly infinite direct product of the space $(X, \mathcal{F}, \mu)$. Define $\widehat{T}: Y \rightarrow Y$ by

$$
\widehat{T}\left(\ldots, x_{-1}, \stackrel{*}{x_{0}}, x_{1}, x_{2}, \ldots\right)=\left(\ldots, T^{-1} x_{-1}, \stackrel{*}{T} x_{0}, T^{-1} x_{1}, T x_{2}, \ldots\right),
$$

where the ${ }^{*}$ indicates the zeroth coordinate.

Suppose now that $S: Y \rightarrow Y$ is the shift map; then $S$ is an ergodic automorphism and it is easy to check that $S \widehat{T}=\widehat{T}^{-1} S$. In fact $S$ is a mixing automorphism.

Note that there are elements of $\mathcal{B}(\widehat{T})$ which are involutions. For example, define $S_{1}: Y \rightarrow Y$ by

$$
S_{1}\left(\ldots, x_{-1}, \stackrel{*}{x}_{0}, x_{1}, x_{2}, \ldots\right)=\left(\ldots, x_{-1}, x_{-2}, \stackrel{*}{x}{ }_{1}, x_{0}, x_{3}, x_{2}, \ldots\right) ;
$$

then $S_{1} \in \mathcal{B}(\widehat{T})$ and $S_{1}^{2}=I$.

(iii) Mathew and Nadkarni [6] defined a continuum of examples, each having a spectrum consisting of a discrete component and a Lebesgue component of multiplicity equal to 2 . It was shown in [2] that each of these transformations $T_{\phi}$ is isomorphic to its inverse, and that every $S \in \mathcal{B}\left(T_{\phi}\right)$ satisfies $S^{4}=I$ and $S^{2} \neq I$. Consequently the results of sections 2 and 3 are applicable. In fact, using the notation of Theorem 3 we have here the situation where $\mathcal{P}_{1}=\{0\}=\mathcal{P}_{2}$ and $L^{2}(X, \mu)=H_{1} \oplus H_{-1}$, with $H_{-1} \neq\{0\}$.

$T_{\phi}:[0,1) \times \mathbb{Z}_{2} \rightarrow[0,1) \times \mathbb{Z}_{2}$ is defined by $T_{\phi}(x, g)=(T x, \phi(x)+g)$ where $T$ is the von Neumann Kakutani adding machine, and $\phi$ is defined using a "random" Toeplitz sequence with " 2 holes", i.e. at the $n$th stage in the construction of the $2^{n}$ levels of $T, \phi$ is left undefined on the $2^{n-1}$ th and top levels of the stack. See [2] for details. 


\section{REFERENCES}

1. A. Fathi, Le groupe de transformations de $[0,1]$ qui preservant la measure de Lebesgue est simple, Isr. J. Math. 29 (1978), 302-308. MR 58:6156

2. G.R. Goodson, A. del Junco, M. Lemańczyk, D.J. Rudolph, Ergodic transformations conjugate to their inverses by involutions, Ergodic Theory and Dynamical Systems 16 (1996), 97-124.

3. A. del Junco, M. Lemańczyk, Generic spectral properties of measure preserving maps and actions, Proc. A.M.S. 115 (1992), 725-736. MR 92i:28017

4. A. Katok, unpublished lecture notes.

5. M. Lemańczyk, Toeplitz- $\mathbb{Z}_{2}$ extensions, Ann. Inst. Henri Poincaŕe 24 (1988), 1-43. MR 90b: 28020

6. J. Mathew, M.G. Nadkarni, A measure preserving transformation whose spectrum has a Lebesgue component of multiplicity two, Bull. Lond. Math. Soc. 16 (1984), 402-406. MR 86b: 28017

7. M. Queffelec, Substitution Dynamical Systems, Spectral Analysis: Lecture Notes in Math., vol. 1294, 1987. MR 89g:54094

8. V.V. Ryzhikov, Representation of transformations preserving Lebesgue measure as a composition of periodic transformations, Mat. Zametky 38 (1985), 860-865; English transl., Math. Notes 38 (1985), 978-981. MR 87b:28018

Department of Mathematics, Towson State University, Towson, Maryland 212047097

E-mail address: e7m2grg@toe.towson.edu

Institute of Mathematics, Nicholas Copernicus University, ul. Chopina 12/18, 87-100 TORUn, POLAND

E-mail address: mlem@mat.uni.torun.pl 